\title{
Effect of IL-1 $\beta$, IL-8, and IL-10 polymorphisms on the development of myocardial infarction
}

\author{
S. Wang ${ }^{1,2 *}$, Y.X. Dai ${ }^{3 *}$, L.L. Chen ${ }^{4}$, T. Jiang ${ }^{5}$, M.Q. Zheng ${ }^{5}$ C.G. Li ${ }^{4}$, \\ Y.P. Chen ${ }^{2}$, W.H. Lin ${ }^{2}$, J.F. Zhang ${ }^{2}$ and J. Jiang ${ }^{1}$ \\ ${ }^{1}$ Department of Cardiology, Second Affiliated Hospital, School of Medicine, \\ Zhejiang University, Hangzhou, China \\ ${ }^{2}$ Department of Cardiology, The First People's Hospital of Wenling, \\ Wenling, China \\ ${ }^{3}$ Shanghai Institute of Cardiovascular Diseases, Zhongshan Hospital, \\ Fudan University, Shanghai, China \\ ${ }^{4}$ Department of Gastroenterology, The First People's Hospital of Wenling, \\ Wenling, China \\ ${ }^{5}$ Central Laboratory, The First People's Hospital of Wenling, Wenling, China \\ *These authors contributed equally to this study. \\ Corresponding author: J. Jiang \\ jiangjun_92@163.com
}

Genet. Mol. Res. 14 (4): 12016-12021 (2015)

Received January 29, 2015

Accepted June 8, 2015

Published October 5, 2015

DOI http://dx.doi.org/10.4238/2015.October.5.14

\begin{abstract}
Myocardial infarction (MI) is currently a leading cause of death worldwide, and is caused by various environmental and genetic factors. We therefore conducted a case-control study to investigate the association between polymorphisms in interleukins IL-1 $\beta$, IL-8, and IL10 and MI risk. This study recruited $260 \mathrm{MI}$ patients and 285 control subjects. Genotyping of IL-1 $\beta+3954 \mathrm{C} / \mathrm{T}$, IL-8 -251T/A, IL-10 - 1082A/G, and IL-10 $-819 \mathrm{C} / \mathrm{T}$ were assessed using the polymerase chain reaction-
\end{abstract}


restriction fragment length polymorphism method. By comparing the risk factors of MI between the case and control groups, we discovered that MI patients were more likely to have smoking and drinking habits, have a history of hypertension and diabetes, have higher triglycerides and low-density lipoprotein cholesterol levels, and a lower high-density lipoprotein cholesterol level $(\mathrm{P}<0.05)$. Unconditional regression analyses showed that subjects carrying the GG genotype of the IL-10 $-1082 \mathrm{~A} / \mathrm{G}$ polymorphism were associated with increased risk of MI, and the OR $(95 \% \mathrm{CI})$ was 2.04 (1.15-3.65). Our study found that the IL-10 -1082A/G polymorphism plays an important role in influencing the development of MI.

Key words: Myocardial infarction; Interleukin factors; Polymorphism

\section{INTRODUCTION}

Myocardial infarction (MI) is a common neurological disease and a leading cause of death worldwide. The etiology of MI is complicated and not completely understood. Several factors are considered important in the development of MI, including genetic and environmental influences (Lopez et al., 2006). The main environmental factors for MI include hypertension, hypercholesterolemia, diabetes, obesity, and smoking (Lopez et al., 2006). Moreover, studies have reported that genetic polymorphisms play an important role in the development of MI (Ianni et al., 2012; Konenkov et al., 2012; Arun Kumar et al., 2015).

The inflammatory mechanism can contribute to the process of MI, and cytokines are the main mediators of the inflammatory response. Inflammation also contributes to ischemic events through the promotion of atherosclerosis (Ross, 1999). Therefore, functional polymorphisms in inflammatory genes could influence the development and outcome of MI.

Previous studies have reported that C-reactive protein and interleukin (IL)-6 are risk factors for cardiovascular disease (Danesh et al., 2000; Hirschfield and Pepys, 2003), and IL-10 and tumor necrosis factor- $\alpha$ are associated with development of cardiovascular outcomes in adults (Kritchevsky et al., 2005). However, few studies have reported the association between $I L-8$ gene polymorphisms and MI risk. Therefore, we conducted this casecontrol study to investigate the association between IL-1 $\beta$, IL-8, and IL-10 polymorphisms and MI risk.

\section{MATERIAL AND METHODS}

\section{Patients}

We recruited $260 \mathrm{MI}$ patients and 285 control subjects. The MI patients were recruited from visitors to the First People's Hospital of Wenling. MI patients were confirmed through computed tomography, magnetic resonance imaging, angiography, or duplex sonography by well-trained physicians. Patients were excluded from our study if they presented accidental or iatrogenic stroke, transient ischemic attack, brain tumors, or other cerebrovascular disorders. The controls were recruited from people who visited our hospital for a general health check- 
up. Controls who had a history of stroke, ischemic heart diseases, neurological diseases, or any severe underlying diseases were excluded from our study. The study was approved by the First People's Hospital of Wenling, and all subjects gave their informed consent prior to inclusion in the study.

The clinical and pathological data were retrospectively collected from medical records. We used a questionnaire that enquired about social and demographic characteristics and risk factors for MI, including gender, age, hypertension, diabetes, cholesterol levels, body mass index, smoking, and physical exercise frequency.

\section{Genotyping analysis}

All study participants were asked to provide $5 \mathrm{~mL}$ venous blood, which was collected in ethylenediaminetetraacetic acid-coated tubes and stored at $-20^{\circ} \mathrm{C}$ until required. Genomic DNA was extracted from peripheral blood lymphocytes and analyzed using a TIANamp Blood DNA Kit (Tiangen Biotech Co., Ltd., Beijing, China). Genotyping of IL-1 $\beta+3954 C / T$, IL-8 $-251 \mathrm{~T} / \mathrm{A}, \mathrm{IL}-10-1082 \mathrm{~A} / \mathrm{G}$, and IL-10 $-819 \mathrm{C} / \mathrm{T}$ were assessed by the polymerase chain reactionrestriction fragment length polymorphism method. Primers and probes of IL- $1 \beta+3954 \mathrm{C} / \mathrm{T}$, IL-8 -251T/A, IL-10 -1082A/G, and IL-10 -819C/T were designed using the Sequenom Assay Design 3.1 software. The cycling program for the polymerase chain reaction was as follows: denaturation at $95^{\circ} \mathrm{C}$ for $10 \mathrm{~min}$, followed by 40 cycles at $95^{\circ} \mathrm{C}$ for $30 \mathrm{~s}, 62^{\circ} \mathrm{C}$ for $30 \mathrm{~s}, 72^{\circ} \mathrm{C}$ for $30 \mathrm{~s}$, and extension at $72^{\circ} \mathrm{C}$ for $10 \mathrm{~min}$. Genotyping was repeated in $5 \%$ of samples to check for accuracy.

\section{Statistical analysis}

Frequencies were used to describe the distribution of categorical variables and median and interquartile range was used for continuous variables. Differences in frequency distributions of the selected demographic variables and risk factors for MI were evaluated between the patients with MI and the control subjects using the chi-square test or Student $t$-test. The Hardy-Weinberg equilibrium for genotype distribution in the controls was tested by a goodness-of-fit chi-square test. Logistic regression was taken to assess the association between IL-1 $\beta+3954 \mathrm{C} / \mathrm{T}$, IL-8 -251T/A, IL-10 -1082A/G, and IL-10 -819C/T polymorphisms and MI risk, and the odds ratios (ORs) and $95 \%$ confidence intervals $(95 \% \mathrm{CIs})$ of the results were determined. $\mathrm{P}<0.05$ was considered a statistically significant difference. Statistical analyses were conducted using SPSS version 16.0 (SPSS, Inc., Chicago, IL, USA).

\section{RESULTS}

The 260 MI patients comprised 204 males and 56 females, and the mean age was $53.81 \pm 9.54$ years. The 285 controls comprised 219 males and 66 females, and the mean age was $53.51 \pm 10.25$ years (Table 1). By comparing the risk factors of MI between the two groups, MI patients were more likely to have a habit of smoking and drinking, have a history of hypertension and diabetes, and have higher triglycerides, total cholesterol and low-density lipoprotein cholesterol levels, and a lower high-density lipoprotein cholesterol level $(\mathrm{P}<0.05)$. 


\section{Table 1. Clinical characteristics of patients.}

\begin{tabular}{|c|c|c|c|c|}
\hline Characteristics & MI cases $(\%)$ & Controls (\%) & $\chi^{2}$ or $t$-test & $P$ value \\
\hline \multicolumn{5}{|l|}{ Age (years) } \\
\hline$<50$ & $93(25.83)$ & $102(35.79)$ & \multirow[t]{2}{*}{0.00} & \multirow[t]{2}{*}{0.99} \\
\hline$\geq 50$ & $167(46.39)$ & $183(64.21)$ & & \\
\hline \multicolumn{5}{|l|}{ Gender } \\
\hline Male & $204(78.46)$ & $219(76.84)$ & \multirow[t]{2}{*}{0.21} & \multirow[t]{2}{*}{0.65} \\
\hline Female & $56(21.54)$ & $66(23.16)$ & & \\
\hline \multicolumn{5}{|l|}{ Smoking status } \\
\hline Never & $124(47.69)$ & $176(61.75)$ & \multirow[t]{2}{*}{10.86} & \multirow[t]{2}{*}{$<0.05$} \\
\hline Current or former & $136(52.31)$ & $109(38.25)$ & & \\
\hline \multicolumn{5}{|l|}{ Drinking status } \\
\hline Never & $99(38.08)$ & $159(55.79)$ & \multirow[t]{2}{*}{17.11} & \multirow[t]{2}{*}{$<0.05$} \\
\hline Current or former & $161(61.92)$ & $126(44.21)$ & & \\
\hline \multicolumn{5}{|l|}{ Hypertension } \\
\hline No & $104(40.00)$ & $190(66.67)$ & \multirow[t]{2}{*}{38.92} & \multirow[t]{2}{*}{$<0.05$} \\
\hline Yes & $156(60.00)$ & $95(33.33)$ & & \\
\hline \multicolumn{5}{|l|}{ Diabetes } \\
\hline No & $150(57.69)$ & $223(78.25)$ & \multirow[t]{2}{*}{26.59} & \multirow[t]{2}{*}{$<0.05$} \\
\hline Yes & $110(42.31)$ & $62(21.75)$ & & \\
\hline Triglycerides (mM) & $4.26 \pm 0.81$ & $3.83 \pm 0.71$ & 6.60 & $<0.05$ \\
\hline Total cholesterol (mM) & $5.06 \pm 1.31$ & $4.78 \pm 1.20$ & 2.60 & $<0.05$ \\
\hline LDL-C (mM) & $3.34 \pm 0.91$ & $2.71 \pm 0.88$ & 8.21 & $<0.05$ \\
\hline HDL-C (mM) & $1.08 \pm 0.29$ & $1.35 \pm 0.53$ & 7.28 & $<0.05$ \\
\hline
\end{tabular}

$\mathrm{MI}=$ myocardial infarction; LDL-C = low-density lipoprotein cholesterol; HDL-C = high-density lipoprotein cholesterol.

The genotype distributions of IL-10 -1082A/G and IL-10 -819C/T were found to be in line with the Hardy-Weinberg equilibrium in the control group, but the IL- $1 \beta+3954 \mathrm{C} / \mathrm{T}$ and IL-8 -251T/A distributions were not.

Unconditional regression analyses showed that subjects carrying the GG genotype of IL-10 - 1082A/G were associated with increased risk of MI, and the OR $(95 \% \mathrm{CI})$ was 2.04 (1.15-3.65) (Table 2). However, we found that IL-1 $\beta+3954 \mathrm{C} / \mathrm{T}$, IL -8-251T/A, and IL-10 $-819 \mathrm{C} / \mathrm{T}$ were not correlated with MI risk.

Table 2. Association between IL-1 $\beta+3954 \mathrm{C} / \mathrm{T}$, IL-8 -251T/A, IL-10 -1082A/G, and IL-10 -819C/T polymorphisms and myocardial infarction (MI) risk.

\begin{tabular}{|c|c|c|c|c|}
\hline Polymorphism & MI cases & Controls & OR $(95 \% \mathrm{CI})^{1}$ & $P$ value \\
\hline \multicolumn{5}{|l|}{$\mathrm{IL}-1 \beta+3954 \mathrm{C} / \mathrm{T}$} \\
\hline $\mathrm{CC}$ & $191(73.46)$ & $218(76.49)$ & 1.0 (Ref) & \\
\hline $\mathrm{CT}$ & $53(20.38)$ & $52(18.25)$ & $1.16(0.74-1.83)$ & 0.49 \\
\hline TT & $16(6.15)$ & $15(5.26)$ & $1.22(0.55-2.72)$ & 0.6 \\
\hline \multicolumn{5}{|l|}{ IL-8 -251T/A } \\
\hline TT & $72(27.69)$ & $89(31.23)$ & 1.0 (Ref) & \\
\hline TA & $113(43.46)$ & $119(41.75)$ & $1.17(0.77-1.79)$ & 0.44 \\
\hline AA & $75(28.85)$ & $77(27.02)$ & $1.20(0.75-1.92)$ & 0.41 \\
\hline \multicolumn{5}{|l|}{ IL-10 $-1082 \mathrm{~A} / \mathrm{G}$} \\
\hline AA & $118(45.38)$ & $155(54.39)$ & 1.0 (Ref) & \\
\hline $\mathrm{AG}$ & $100(38.46)$ & $103(36.14)$ & $1.28(0.87-1.87)$ & 0.26 \\
\hline GG & $42(16.15)$ & $27(9.47)$ & $2.04(1.15-3.65)$ & $<0.05$ \\
\hline \multicolumn{5}{|l|}{ IL-10 -819C/T } \\
\hline $\mathrm{CC}$ & $89(34.23)$ & $108(37.89)$ & 1.0 (Ref) & \\
\hline $\mathrm{CT}$ & $119(45.77)$ & $126(44.21)$ & $1.15(0.77-1.70)$ & 0.48 \\
\hline TT & $52(20.00)$ & $51(17.89)$ & $1.24(0.75-2.05)$ & 0.38 \\
\hline
\end{tabular}

${ }^{1}$ Adjusted for gender, age, smoking and drinking status, hypertension, diabetes, triglycerides, total cholesterol, lowdensity lipoprotein cholesterol (LDL-C), and high-density lipoprotein cholesterol (HDL-C). 


\section{DISCUSSION}

Cytokines are modulators of immune responses, and the balance between proinflammatory and anti-inflammatory stimuli plays an important role in the development of atherosclerosis. It is reported that locally higher secretion and concentrations of proinflammatory cytokines can cause severe damage to the epithelium of blood vessels and the surrounding tissues, and cytokines from lymphocytes within the inflamed site can induce more damage. Therefore, genetic polymorphisms of the functional cytokines are associated with increased risk of vascular lesions.

Our study found that the IL-10 -1082A/G polymorphism may be associated with MI risk. Previous studies have reported that the IL-10 $-1082 \mathrm{~A} / \mathrm{G}$ polymorphism is associated with the risk of many blood vessel-related diseases, such as ischemic stroke (IS), deep venous thrombosis, and sepsis (Ouyang et al., 2013; Jin et al., 2014; Tang et al., 2014). Jin et al. (2014) conducted a meta-analysis of seven studies and found that the IL-10 -1082A/G polymorphism is associated with IS susceptibility in Asians and the $-1082 \mathrm{~A}$ allele may increase risk of IS in Asians. Tang et al. (2014) conducted a case-control study in a Chinese population and found that the IL-10 $-1082 \mathrm{~A} / \mathrm{G}$ polymorphism is associated with risk of deep venous thrombosis. Another meta-analysis study of 11 studies investigated the association between the IL-10 $-1082 \mathrm{~A} / \mathrm{G}$ polymorphism and susceptibility to sepsis, and it revealed that the IL-10 $-1082 \mathrm{~A} / \mathrm{G}$ polymorphism has an association with susceptibility to sepsis (Ouyang et al., 2013). For the association between the IL-10 -1082A/G polymorphism and cardiovascular disease, one previous meta-analysis with 5006 patients and 3968 controls found that the A allele of the IL-10 -1082A/G polymorphism is associated with increased risk of cardiovascular disease (Wang et al., 2012). Few studies have reported the association between the IL-10 -1082A/G polymorphism and MI risk. Therefore, further well-designed studies are required to confirm the association between the IL-10 -1082A/G polymorphism and MI risk.

Several limitations in this study should be addressed. First, the case subjects and controls were recruited from one hospital, which may not have accurately represented the general population. Moreover, the genetic distributions of IL-1 $\beta+3954 \mathrm{C} / \mathrm{T}$ and IL-8 -251T/A were not in Hardy-Weinberg equilibrium. Selection bias may have existed in our study. Second, the sample size of our study was relatively small, especially in the case subjects, which may have limited the statistical power to determine the difference between the groups. Third, some interaction between the IL-1 $\beta$, IL-8, and IL-10 gene polymorphisms and environmental factors may have existed in our study, but we did not investigate their association. Therefore, further large sample size studies are required to verify the association between the IL-1 $\beta$, IL-8, and IL-10 gene polymorphisms and risk of MI.

In conclusion, our study found that the IL-10 $-1082 \mathrm{~A} / \mathrm{G}$ polymorphism plays an important role in influencing the development of MI. Further well-designed studies and studies with large sample sizes are required to confirm our results.

\section{Conflicts of interest}

The authors declare no conflict of interest. 


\section{REFERENCES}

Arun Kumar AS, Kumar SS, Umamaheswaran G, Kesavan R, et al. (2015). Association of CYP2C8, CYP2C9 and CYP2J2 gene polymorphisms with myocardial infarction in South Indian population. Pharmacol. Rep. 67: 97-101.

Danesh J, Whincup P, Walker M, Lennon L, et al. (2000). Low grade inflammation and coronary heart disease: prospective study and updated meta-analyses. BMJ 321: 199-204.

Hirschfield GM and Pepys MB (2003). C-reactive protein and cardiovascular disease: new insights from an old molecule. QJM 96: 793-807.

Ianni M, Callegari S, Rizzo A, Pastori P, et al. (2012). Pro-inflammatory genetic profile and familiarity of acute myocardial infarction. Immun. Ageing 9: 14.

Jin J, Li W, Peng L, Chen J, et al. (2014). Relationship between interleukin-10 -1082A/G polymorphism and risk of ischemic stroke: a meta-analysis. PLoS One 9: e94631.

Konenkov VI, Shevchenko AV, Prokof'ev VF and Maksimov VN (2012). Complex of genotypes of cytokines as a genetic factor of risk of development of myocardial infarction of in Europien population of Russia men. Kardiologiia 52: $22-29$.

Kritchevsky SB, Cesari M and Pahor M (2005). Inflammatory markers and cardiovascular health in older adults. Cardiovasc. Res. 66: 265-275.

Lopez AD, Mathers CD, Ezzati M, Jamison DT, et al. (2006). Global and regional burden of disease and risk factors, 2001: systematic analysis of population health data. Lancet 367: 1747-1757.

Ouyang L, Lv YD, Hou C, Wu GB, et al. (2013). Quantitative analysis of the association between interleukin-10 1082A/G polymorphism and susceptibility to sepsis. Mol. Biol. Rep. 40: 4327-4332.

Ross R (1999). Atherosclerosis - an inflammatory disease. N. Engl. J. Med. 340: 115-126.

Tang B, Chen YK, Luo WJ, Fu J, et al. (2014). Association between interleukin-10 -1082A/G, -819C/T and -592C/A polymorphisms with deep venous thrombosis. Hum. Immunol. 75: 203-207.

Wang Y, Zheng J, Liu P, Yu X, et al. (2012). Association between the interleukin 10-1082G $>$ A polymorphism and coronary heart disease risk in a Caucasian population: a meta-analysis. Int. J. Immunogenet. 39: 144-150. 